\title{
Self-Assembly and Two-Dimensional Spontaneous Resolution of Cyano-Functionalized [7]Helicenes on $\mathrm{Cu}(111)^{* *}$
}

\author{
Meike Stöhr,* Serpil Boz, Michael Schär, Manh-Thuong Nguyen, Carlo A. Pignedoli, \\ Daniele Passerone, * W. Bernd Schweizer, Carlo Thilgen, Thomas A. Jung, * and \\ François Diederich*
}

In memoriam Emanuel Vogel

Effective control of chirality in supramolecular systems is an important challenge, for example in the fields of (heterogeneous) asymmetric catalysis ${ }^{[1]}$ and liquid crystals. ${ }^{[2]}$ The spontaneous resolution of a racemic compound into a conglomerate of enantiomeric crystals is based on a preference of molecules to make contacts with neighbors of the same chirality sense through supramolecular interactions. ${ }^{[3]}$ Although considerable progress has been made in the prediction of crystal structures, ${ }^{[4]}$ the occurrence of spontaneous resolution in the course of the formation of crystals in three dimensions (3D) still lacks reliable predictability. Therefore, scanning tunneling microscopy (STM) studies of the formation of $2 \mathrm{D}$ conglomerates from surface-supported racemic mixtures of molecules provide valuable insight into the phenomenon of spontaneous resolution ${ }^{[3,5]}$ and the underlying intermolecular interactions.

Helicity is a fundamental element of molecular chirality ${ }^{[6]}$ and supramolecular interactions between helices are of utmost importance in molecular biology. ${ }^{[7]}$ The carbonbased $[n]$ helicenes, ${ }^{[8]}$ ortho-fused polycyclic aromatic hydro-

[*] Prof. M. Stöhr

Zernike Institute for Advanced Materials, University of Groningen Nijenborgh 4, 9747 AG Groningen (The Netherlands)

E-mail:m.a.stohr@rug.nl

Dr. S. Boz, Prof. T. A. Jung

Department of Physics, University of Basel

Klingelbergstrasse 82, 4056 Basel (Switzerland)

E-mail: thomas.jung@psi.ch

Dr. M. Schär, Dr. W. B. Schweizer, Prof. C. Thilgen, Prof. F. Diederich Laboratorium für Organische Chemie, ETH Zürich

Wolfgang-Pauli-Strasse 10, 8093 Zürich (Switzerland)

E-mail: diederich@org.chem.ethz.ch

M.-T. Nguyen, Dr. C. A. Pignedoli, Dr. D. Passerone

Empa, Swiss Federal Laboratories for Materials Science and

Technology, nanotech@surfaces laboratory

Überlandstrasse 129, 8600 Dübendorf (Switzerland)

E-mail: Daniele.Passerone@empa.ch

$\left[{ }^{*} \cdots\right]$ This work was supported by the European Union through the Marie Curie Research Training Network PRAIRIES (contract MRTN-CT2006-035810), the Swiss National Science Foundation, the NCCR "Nanoscale Science", and the Wolfermann-Nägeli-Stiftung. The Swiss National Supercomputing Centre (CSCS) is acknowledged for the use of computer time. We thank S. Schnell for his support with building and maintaining the experimental infrastructure.

Supporting information for this article is available on the WWWW under http://dx.doi.org/10.1002/anie.201102627. carbons with $n \geq 5$ angularly arranged benzene rings, are a prototypical example of cylindrical molecular helices. In particular, the adsorption of [7]helicene on $\mathrm{Cu}(111)$ has been at the focus of research attempting to unveil the principles of self-assembly for these chiral hydrocarbons. ${ }^{[9]} \mathrm{A}$ racemic mixture of heptahelicene was shown to form zigzag-type rows of alternating $P$ - and $M$-configured molecules. ${ }^{\left[{ }^{[d]}\right]}$ These rows assembled into "2D racemate" type chiral domains, the underlying intermolecular interactions being based on nondirectional van der Waals forces. Up to now, no spontaneous resolution of enantiomers has been observed for racemic helicenes adsorbed on surfaces. This contrasts with the 3D crystallization behavior of many unsubstituted helicenes which form conglomerates of (micro)crystals, often featuring microtwinning or lamellar twinning. ${ }^{[\mathrm{a}-\mathrm{c}]}$ The title compound, 6,13-dicyano[7]helicene (1, Scheme 1 and Figure $1 \mathrm{a})$, on the other hand, crystallized as solvent-free racemate from a solution of $( \pm)-\mathbf{1}$ in $\mathrm{CH}_{2} \mathrm{Cl}_{2}$, and as the solvate (+)-(P)$\mathbf{1} \cdot \mathrm{CH}_{2} \mathrm{Cl}_{2}$ from a solution of pure $(+)-(P)-\mathbf{1}$ (see the Supporting Information).

Here, we present a combined STM and DFT (density functional theory) study for the adsorption of a [7]helicene functionalized with two cyano groups (1) on $\mathrm{Cu}(111)$. We demonstrate the formation of enantiopure domains in which homochiral molecules are assembled either in the form of "dimers" or "tetramers". Through atomistic simulation, we understand the role of supramolecular interactions in this diastereoselective self-assembly process on the copper surface. Indeed, our experimental and theoretical findings show that supramolecular synthons based on $\mathrm{CN} \cdots \mathrm{HC}(\mathrm{Ar})$ hydrogen bonding and dipolar $\mathrm{CN} \cdots \mathrm{CN}$ interactions, both of which are well known from $3 \mathrm{D}$ crystals ${ }^{[10]}$ and $2 \mathrm{D}$ surface architectures, ${ }^{[11]}$ play also a role in the conglomerate-type $2 \mathrm{D}$ selfassembly (spontaneous resolution) of cyanohelicenes.

A versatile method was elaborated for the preparation of pure enantiomers of 6,13-dicyano[7]helicene $((P)$-1 and $(M)$ 1, Scheme 1). It includes the photocyclodehydrogenation of stilbene-type precursors ${ }^{[12]} \mathbf{2}$ as the key, helicene-forming step as well as a chromatographic resolution of the resulting helicene derivative 3. Distilbene $\mathbf{2}$ is available in three steps from naphthalene-2,3-dimethanol ${ }^{[13]}$ (see Scheme 1 in the Supporting Information). Taking advantage of the directing effect of the Br substituent ("bromine-auxiliary" strategy), ${ }^{[14]}$ helicene precursor $\mathbf{2}$ was regioselectively converted into racemic [7] helicene $( \pm)-\mathbf{3}$ by photocyclodehydrogenation 


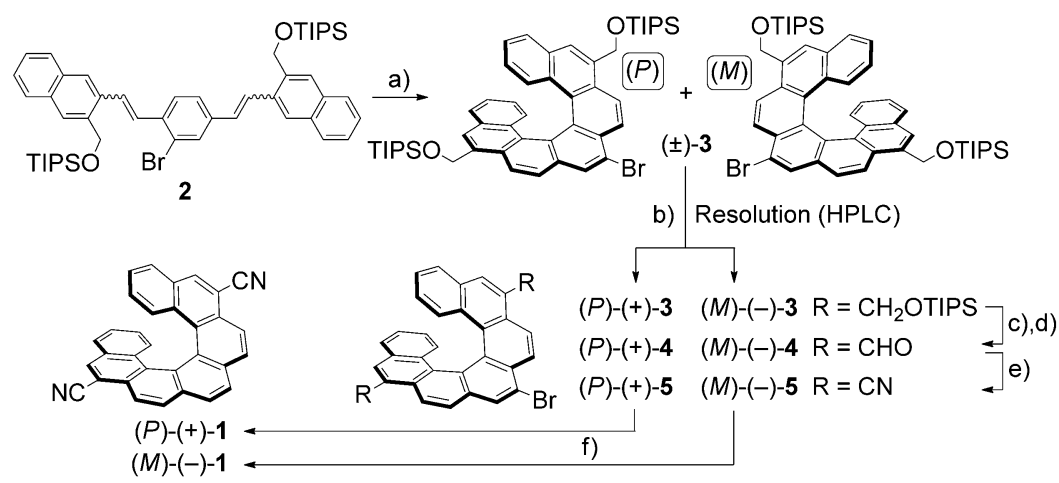

Scheme 1. a) $h v$ (Ga-doped high-pressure Hg lamp), $I_{2},( \pm)$-propylene oxide, PhMe, RT, $19 \mathrm{~h}, 73 \%( \pm)-3$; b) (S,S)-Whelk-O1 CSP (Regis Technologies); c) $n B u_{4} N F, T H F, R T, 1$; d) PCC, $\mathrm{CH}_{2} \mathrm{Cl}_{2}$, molecular sieves $3 \AA$, RT, $1 \mathrm{~h}, 85 \%(P)-(+)-4$ (two steps); e) 1. $\mathrm{H}_{2} \mathrm{NOH} \cdot \mathrm{HCl}$, pyridine, $\mathrm{H}_{2} \mathrm{O}, 1.5 \mathrm{~h}, \mathrm{RT}$; 2. DCC, $\mathrm{Et}_{3} \mathrm{~N}, \mathrm{CuSO}_{4} \cdot 5 \mathrm{H}_{2} \mathrm{O}, \mathrm{CH}_{2} \mathrm{Cl}_{2}, 50^{\circ} \mathrm{C}$, 20 h, 89\% (P)-(+)-5; f) [Pd( $\left.\left(\mathrm{PPh}_{3}\right)_{4}\right], \mathrm{K}_{2} \mathrm{CO}_{3}, n \mathrm{BuOH}, \mathrm{PhMe}, 60^{\circ} \mathrm{C}, 16 \mathrm{~h}, 98 \%(P)-(+)-1$. $\mathrm{CSP}=$ chiral stationary phase, $\mathrm{PCC}=$ pyridinium chlorochromate, $\mathrm{DCC}=\mathrm{N}, \mathrm{N}^{\prime}$-dicyclohexyl carbodiimide, TIPS $=$ triisopropylsilyl. orientation was observed for unsubstituted [7]helicene at submonolayer coverage in recent STM studies $^{[9,18]}$ and is expected because of considerable interactions between the extended $\pi$ system of the polycyclic aromatic hydrocarbon and the metallic substrate, a tilted "out-of-plane" arrangement $\left((43 \pm 5)^{\circ}\right.$ off the surface) was found for pristine $(P)$-[7]helicene on $\mathrm{Ni}(100)$ by NEXAFS (near-edge X-ray absorption fine structure) measurements at monolayer coverage. ${ }^{[19]}$ And very recently, an edge-on ("standing upright") orientation was reported for a carboxyhelicene adsorbed on calcite. ${ }^{[20]}$

Through a combination of experimental and theoretical investigations, we first determined the adsorption geometry for individual helicene molecules $\mathbf{1}$ on $\mathrm{Cu}(111)$. This is relevant for the later discussion on the intermolecular interactions for the different a)

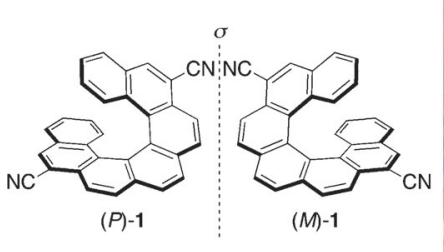

b)

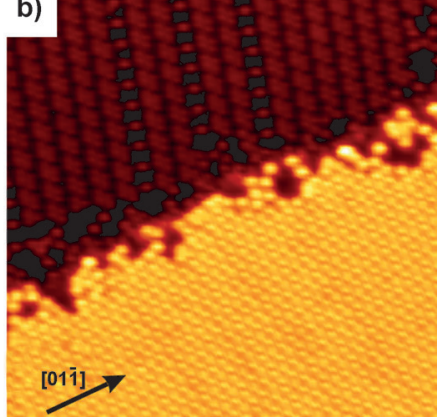

Figure 1. a) Molecular structures of the two enantiomers of 6,13-dicyano[7] helicene, $(P)-1$ and $(M)-1$. b) Overview STM image $\left(43 \times 43 \mathrm{~nm}^{2}, 77 \mathrm{~K}\right)$ of $(P)-1$, showing a dimeric (bottom) next to a tetrameric (top) arrangement. Note that a $\mathrm{Cu}$ step edge runs from the lower left to the upper right.

according to Katz and co-workers. ${ }^{[14 a]}$ The highly soluble TIPS-protected [7]helicene-dimethanol $( \pm)$-3 was efficiently resolved into the enantiomers by HPLC on an $(S, S)$-WhelkO1 chiral stationary phase (see Figure 8 in the Supporting Information). Desilylation of (+)-3, followed by oxidation of the resulting diol, afforded dialdehyde $(+)-\mathbf{4}$. It was transformed into dinitrile $(+)-5$ by a mild one-pot conversion consisting of oxime formation and subsequent dehydration. ${ }^{[15]}$ Final debromination to $(+) \mathbf{- 1}$ was achieved in almost quantitative yield by palladium-catalyzed proto-dehalogenation. ${ }^{[16]}$ The other dicyanohelicene enantiomer, (-)-1, was prepared from pure $(-)-\mathbf{3}$ by the same sequence. The absolute configuration of the final products was unequivocally assigned as (+)-( $(P)-\mathbf{1}$ and $(-)-(M)-\mathbf{1}$ by comparison of the ECD (electronic circular dichroism) spectra (see Figure 9 in the Supporting Information) to experimental and calculated ECD data of similar helicenes. ${ }^{[17]}$

It is important, for the following discussion, to establish the exact adsorption geometry of a single dicyanohelicene molecule 1 on $\mathrm{Cu}(111)$. Although a face-on ("lying flat") patterns observed. With our DFT scheme, we assessed two different adsorption geometries (see the Supporting Information): the face-on orientation turned out to be $0.7 \mathrm{eV}$ more stable than the edge-on geometry, and the corresponding simulated STM images are in good agreement with the experimental measurements (see Figure 15 in the Supporting Information) since the signature of the face-on molecule is present in both.

After adsorption of $(P)-\mathbf{1}$ on $\mathrm{Cu}(111)$ at coverages $\leq 1$ ML (monolayer), well-ordered supramolecular assemblies were observed by STM under ultrahigh-vacuum (UHV) conditions. At coverages of less than $0.8 \mathrm{ML}$, two different arrangements coexist: a dimeric (Figure 1b, bottom) and a less compact tetrameric phase (Figure $1 \mathrm{~b}$, top). The packing density of the latter is approximately 0.73 molecules nm ${ }^{-2}$, whereas that of the dimeric phase is higher, accommodating 0.84 molecules $\mathrm{nm}^{-2}$. At increasing coverage, the denser structure prevails, and close to $1 \mathrm{ML}$, the tetrameric arrangement vanishes completely in favor of the dimeric phase.

Adsorption of the other enantiomer, $(M)-\mathbf{1}$, on $\mathrm{Cu}(111)$ leads to the development of the same coverage-dependent structures. The angle between the symmetry directions of the overlayer and those of the underlying $\mathrm{Cu}$ substrate takes the same absolute value, while the rotational direction is different for the two enantiomers. This is also reflected by the observation that the structures formed by $(P)-\mathbf{1}$ and $(M)-\mathbf{1}$ are mirror images (Figure 2). The dimeric arrangement is commensurate with the $\mathrm{Cu}$ substrate (see the Supporting Information). Consequently, the dimeric pattern leads to the appearance of rotational domains which meet at the same angle $\left(60^{\circ}\right)$ as the principal directions of the $\mathrm{Cu}$ substrate (Figure $3 \mathrm{a}$ ). In essence, the chirality of the molecular building block translates into a chiral motif (either dimeric or tetrameric) on the surface.

When racemic dicyanohelicene $( \pm)-\mathbf{1}$ was deposited on $\mathrm{Cu}(111)$, tetrameric and dimeric structures again formed. It is important to note that, again, exclusively enantiopure 

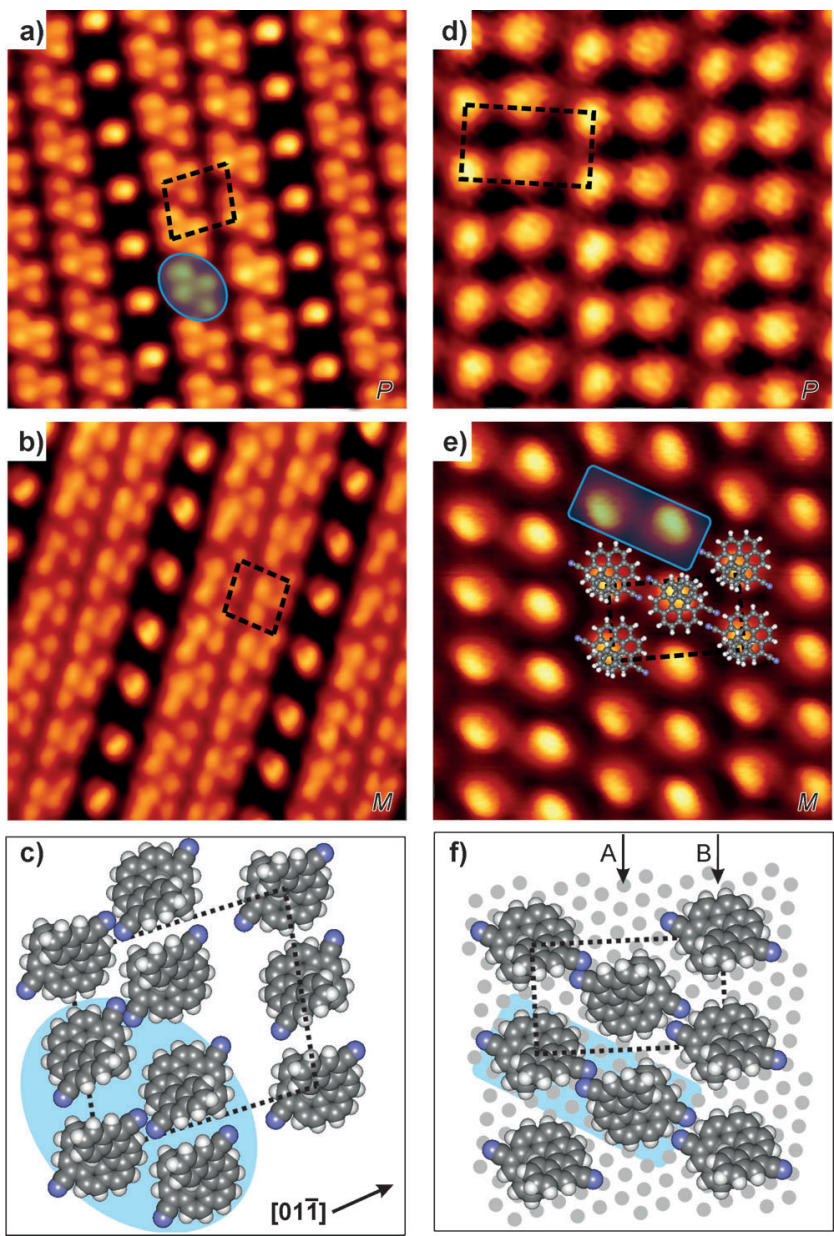

Figure 2. a), b) STM images $\left(15 \times 15 \mathrm{~nm}^{2}, 77 \mathrm{~K}\right)$ of the tetrameric arrangement of enantiopure $(P)-1$ and $(M)$-1, respectively. c) Tentative atomistic model for the arrangement of $(P)-1$. A tetrameric unit is highlighted by a blue ellipse in (a) and (c). The arrow in (c) indicates the high-symmetry direction of the underlying Cu substrate. d), e) STM images $\left(6 \times 6 \mathrm{~nm}^{2}, 77 \mathrm{~K}\right)$ of the dimeric arrangement of enantiopure $(P)-1$ and $(M)-1$, respectively. $f$ ) Atomistic model for the arrangement of $(M)-1$ based on STM and LEED (low-energy electron diffraction) data, which shows alternating $A$ and $B$ rows. A dimeric unit is highlighted by a blue rectangle in (e) and ( $f$ ). The unit cells are marked by black dashed tetragons. The arrangements of $(P)-1$ and $(M)-1$ are mirror-symmetric in both the tetrameric and dimeric cases

domains were detected, consisting of either $(P)-\mathbf{1}$ or $(M)-\mathbf{1}$. Since separate adsorption of enantiomers results in mirrorimage phases (see above), it can be concluded that the enantiopure domains observed after adsorption of $( \pm)-\mathbf{1}$ originate from a spontaneous resolution of the racemic mixture adsorbed on $\mathrm{Cu}(111)$ (see also the LEED measurements in the Supporting Information). In Figure 3b, the upper domain consists of pure $(M) \mathbf{- 1}$ and is separated by a mirror domain boundary from the lower domain composed of pure $(P) \mathbf{- 1}$. The angle between dimeric units of the $(P)-\mathbf{1}$ and $(M)-\mathbf{1}$ domain is about $21.9^{\circ}$ (see the azure rectangles in Figure $3 \mathrm{~b}$ ). This value differs from that found for dimeric units of rotational domains $\left(60^{\circ}\right.$; Figure $\left.3 \mathrm{a}\right)$. Moreover, the angle between the principal $\mathrm{Cu}$ directions and the shorter unit cell vector of the molecular overlayer amounts to $10.9^{\circ}$ (see the

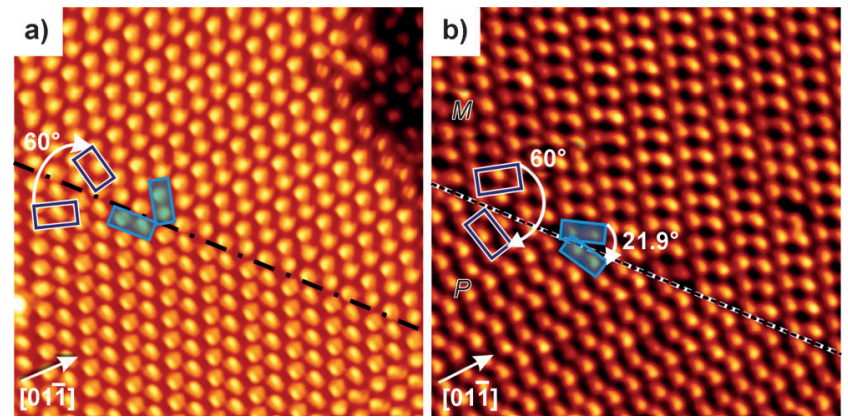

Figure 3. a) STM image $\left(20 \times 20 \mathrm{~nm}^{2}, 77 \mathrm{~K}\right)$ of the dimeric arrangement of $(M)-1$. Two rotational domains adjoin each other at an angle of $60^{\circ}$. b) STM image $\left(20 \times 20 \mathrm{~nm}^{2}, 77 \mathrm{~K}\right)$ of the dimeric arrangement resulting from adsorption of racemic $( \pm)-1$. Two mirror-image, enantiopure domains arise from spontaneous resolution; the top phase is composed of $(M)-1$, the bottom phase of $(P)-1$. The domain boundaries are marked by a black dashed line, the unit cells by dark blue rectangles, the relative arrangement of dimers belonging to different domains by an azure rectangle, and the rotational angle between unit cells of neighboring domains by a curved white arrow. In (b), the rotational angle between dimers of adjacent domains is also indicated by a curved white arrow. The sizes of the various domains generally parallel those of the $\mathrm{Cu}(111)$ terraces, and the number of coexisting domains per terrace decreases with increasing coverage. ${ }^{[21]}$

Supporting Information) which is half the value of $21.9^{\circ}$. It can thus be concluded that the two domains of Figure $3 \mathrm{~b}$ consist of different enantiomers resulting from a spontaneous resolution of $( \pm)-\mathbf{1}$, and that the self-assembly of the chiral dicyanohelicene is diastereoselective. The arrangement of homochiral molecules into dimers, tetramers, and entire enantiopure domains must be energetically favored over that of heterochiral species.

To corroborate our experimental findings, DFT calculations were performed with periodic boundary conditions in the planar directions. The present system involves hydrogen and chemical bonding, which are well-described by standard gradient corrected schemes and dispersive interactions. To account for van der Waals effects we used the correction scheme proposed by Grimme. ${ }^{[22]}$ In spite of its simplicity, it has proven to be very effective not only in the case of pure physisorption but also where chemical interactions play an important role, giving good agreement for the adsorption energies. ${ }^{[23]}$ As input for the calculations, the information obtained from the LEED measurements (see Figures 1 and 2 in the Supporting Information) was used: The unit cell contains two molecules, has rectangular symmetry, and a size of $20.29 \times 11.70 \AA^{2}$, and the lattice vectors define an angle of $90^{\circ}$. Starting from the experimental observation that the dimeric structure consists of alternating $\mathrm{A}$ and $\mathrm{B}$ rows (indicated in Figure 2f), different models were built for a supercell of two molecules (see the Supporting Information), and the atomic positions were optimized in vacuum. Only the model displayed in Figure $2 \mathrm{f}$ (= model E in Figure 14 in the Supporting Information) reproduces, in the calculations, the experimentally observed antiparallel orientation of two molecules forming a dimer. We computed STM images within the Tersoff-Hamann approximation (with application of a Gaussian smearing of $2 \AA$ ) and compared them with the 

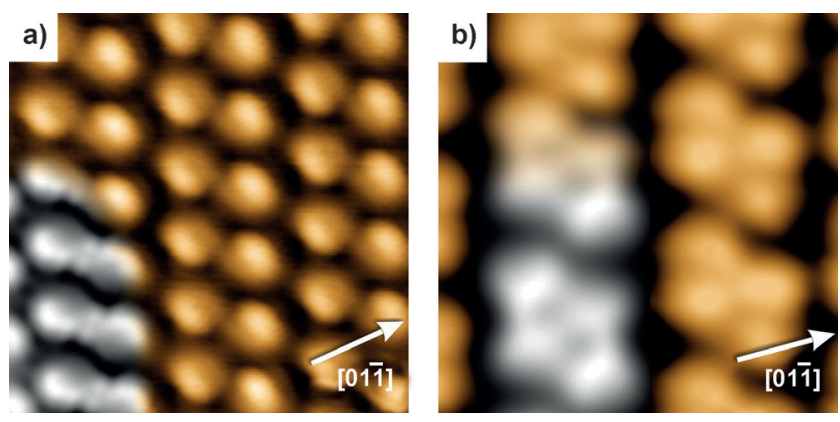

Figure 4. Comparison between experimentally measured (color) and simulated (gray-scale) STM images $\left(6 \times 6 \mathrm{~nm}^{2}\right)$. a) Dimeric arrangement of $(M)-1$ and b) tetrameric arrangement of $(P)-1$. The simulated STM images are based on the models depicted in Figure $2 \mathrm{c}$ and $\mathrm{f}$.

experimental measurements. An excellent agreement is obtained, as can be seen in Figure $4 \mathrm{a}$ (in the Supporting Information the raw STM simulation without Gaussian smearing is shown). The pattern is stabilized by intermolecular antiparallel dipole-dipole interactions between the cyano groups of neighboring molecules, by the interaction between the electric polarizations induced by moleculesurface interactions, and by hydrogen bonding between the cyano groups and hydrogen atoms of neighboring molecules. The STM images computed for the corresponding racemic mixture provide a qualitatively different pattern, as shown in the Supporting Information.

In the tetrameric phase, the two central molecules of a tetramer (highlighted by a blue oval in Figure 2a,c) exhibit the same intermolecular interactions as a dimer (antiparallel dipolar coupling of the cyano groups and $\mathrm{CN} \cdots \mathrm{H}(\mathrm{Ar})$ hydrogen bonding). The outer two helicenes interact with the central ones through hydrogen bonding between a cyano group and an aryl hydrogen atom of a central molecule. Individual tetramers interact with each other through antiparallel dipolar coupling in such a way that rows of tetramers are formed. In this case, too, the agreement between DFT calculations and experimental data (Figure $4 \mathrm{~b}$ ) is very good.

Another question that may be answered by an atomistic simulation concerns the origin of the observed spontaneous resolution of the enantiomers of $\mathbf{1}$ (Figure $3 \mathrm{~b}$ ). We tested the possibility of obtaining dimeric structures that are not enantiomerically pure: if the unit cell is formed by a dimer of molecules with opposite chirality sense, the relative positions of the $\mathrm{CN}$ groups and the nearest hydrogen atoms in the neighboring molecules are not as favorable for hydrogen bonding as it is the case in the homochiral model (Figure $2 \mathrm{f}$ and Figure 15 in the Supporting Information), and the stability of the structure is decreased by $0.1 \mathrm{eV}$ (in vacuum).

This difference alone would not justify the observed diastereoselective self-assembly of homochiral dicyanohelicenes. However, we found that a possible reason for the spontaneous enantiomer separation is the polarization induced in the surface-bound helicene. Indeed, in the gas phase, the molecule has a negligible intrinsic dipole moment, whereas upon adsorption on the $\mathrm{Cu}$ surface, it receives a small amount of charge from the latter (ca. 0.1 electron) and, more importantly, a substrate-induced polarization eventuates, giving rise to a dipole moment of more than 3 Debye on the isolated molecule, becoming 4.3 Debye per molecule in the dimeric phase.

The result of such polarization distributions in an ordered monolayer, for example, the dimeric phase, can be very different for the racemic and the enantiopure case. Indeed, we verified that an enantiopure dimeric phase has a completely different distribution of the induced charge with respect to a racemic phase, as documented, for example, by the distribution of induced dipoles in the lattice (see the Supporting Information). Concerning the electrostatic energy, a full comparison including higher order multipoles would be necessary; therefore we fully optimized the two structures on the surfaces with DFT and we found that the enantiopure phase is more stable than the racemic one by $0.11 \mathrm{eV} / \mathrm{cell}$, even in the presence of the substrate. Interestingly, the bare dipolar interaction energy would point in the other direction, making the racemic phase more stable. However, an interplay between electrostatic effects of higher order, substrate and quantum effects (such as the non-electrostatic part of hydrogen bonding) makes up the computed ab initio result.

In conclusion, we provide the first example of the 2D spontaneous resolution, on $\mathrm{Cu}(111)$, of a racemic mixture of helicenes into long-range-ordered, fully segregated domains of pure enantiomers (2D conglomerate). Upon adsorption of 6,13-dicyano[7]helicene on $\mathrm{Cu}(111)$, concurrent phases based on dimers (denser structure) and tetramers were observed by UHV-STM. Corroborated by DFT calculations, the selfassociation relies on supramolecular synthons based on both $\mathrm{CN} \cdots \mathrm{HC}(\mathrm{Ar})$ hydrogen bonding and dipolar $\mathrm{CN} \cdots \mathrm{CN}$ interactions. The adsorption of enantiomeric helicenes affords phases with mirror-image patterns. In contrast, the adsorption of racemic dicyanohelicene leads to a conglomerate of enantiopure domains which means that the assembly of homochiral molecules is favored over that of heterochiral species. Notably, this spontaneous resolution behavior distinguishes the present case of dicyano[7]helicene from that of unsubstituted [7] helicene. ${ }^{[9 \mathrm{~d}]}$ A possible explanation, at the atomistic level, for this diastereoselective 2D assembly are more favorable interactions between the appreciable molecular dipoles resulting mainly from a substrate-induced polarization, and a higher number of $\mathrm{CN} \cdots \mathrm{HC}(\mathrm{Ar})$ intermolecular hydrogen bonds in the ordered associates of homochiral as opposed to heterochiral dicyanohelicenes.

\section{Experimental Section}

Measurements were carried out in a UHV system consisting of two chambers (one for sample preparation and one for characterization, base pressure: $1 \times 10^{-10} \mathrm{mbar}$ ) or a home-built room-temperature UHV system consisting of five chambers. Low-temperature STM experiments were carried out at $77 \mathrm{~K}$. Typical scanning parameters were $\approx 1.3 \mathrm{~V}$ sample bias and $\approx 20 \mathrm{pA}$ tunneling current. A (111)oriented $\mathrm{Cu}$ single crystal was used as substrate for the molecular films. It was cleaned prior to use by cycles of sputtering with $\mathrm{Ar}^{+}$ions and subsequent annealing at $870 \mathrm{~K}$. Molecules of $\mathbf{1}$ were deposited on the substrate by thermal evaporation from a commercial evaporator 
(Kentax UHV equipment) at $\approx 180^{\circ} \mathrm{C}$. The deposition rate was controlled by means of a quartz crystal microbalance.

Received: April 15, 2011

Revised: August 9, 2011

Published online:

Keywords: chirality - helicenes - scanning probe microscopy spontaneous resolution - surface-confined self-assembly

[1] R. Raval in Nanostructured Catalysts (Eds.: S. L. Scott, C. M. Crudden, C. W. Jones), Springer, New York, 2003, pp. 179-193.

[2] R. Eelkema, B. L. Feringa, Org. Biomol. Chem. 2006, 4, 3729 3745.

[3] L. Pérez-García, D. B. Amabilino, Chem. Soc. Rev. 2002, 31, $342-356$.

[4] a) M. A. Neumann, F. J. J. Leusen, J. Kendrick, Angew. Chem. 2008, 120, 2461-2464; Angew. Chem. Int. Ed. 2008, 47, $2427-$ 2430; b) S. M. Woodley, R. Catlow, Nat. Mater. 2008, 7, 937 - 946.

[5] a) Q. Chen, N. V. Richardson, Annu. Rep. Prog. Chem. Sect. C 2004, 100, 313 - 347; b) A. Kühnle, T. R. Linderoth, B. Hammer, F. Besenbacher, Nature 2002, 415, 891-893; c) M. Lingenfelder, G. Tomba, G. Costantini, L. C. Ciacchi, A. De Vita, K. Kern, Angew. Chem. 2007, 119, 4576-4579; Angew. Chem. Int. Ed. 2007, 46, 4492-4495; d) K.-H. Ernst, Top. Curr. Chem. 2006, 265 209-252; e) A. G. Mark, M. Forster, R. Raval, Tetrahedron: Asymmetry 2010, 21, 1125-1134; f) W. Mamdouh, H. Uji-i, A Gesquière, S. De Feyter, D. B. Amabilino, M. M. S. AbdelMottaleb, J. Veciana, F. C. De Schryver, Langmuir 2004, 20 , 9628-9635.

[6] R. S. Cahn, C. Ingold, V. Prelog, Angew. Chem. 1966, 78, 385 415; Angew. Chem. Int. Ed. Engl. 1966, 5, 413-447.

[7] R. M. Epand, The Amphipathic Helix, CRC, Boca Raton, 1993.

[8] a) W. H. Laarhoven, W. J. C. Prinsen, Top. Curr. Chem. 1984, 125, $63-130$; b) R. H. Martin, Angew. Chem. 1974, 86, 727-738; Angew. Chem. Int. Ed. Engl. 1974, 13, 649-660; c) R. H. Martin, M. J. Marchant, Tetrahedron 1974, 30, 343-345; d) I. Starý, I. G. Stará in Strained Hydrocarbons-Beyond the van't Hoff and Le Bel Hypothesis (Ed.: H. Dodziuk), Wiley-VCH, Weinheim, 2009, pp. 166-201.

[9] a) K.-H. Ernst, Y. Kuster, R. Fasel, M. Müller, U. Ellerbeck, Chirality 2001, 13, 675-678; b) R. Fasel, A. Cossy, K.-H. Ernst, F. Baumberger, T. Greber, J. Osterwalder, J. Chem. Phys. 2001 115, 1020-1027; c) R. Fasel, M. Parschau, K.-H. Ernst, Angew. Chem. 2003, 115, 5336-5339; Angew. Chem. Int. Ed. 2003, 42, 5178-5181; d) R. Fasel, M. Parschau, K.-H. Ernst, Nature 2006 , $439,449-452$.
[10] a) G. R. Desiraju, Angew. Chem. 1995, 107, 2541-2558; Angew. Chem. Int. Ed. Engl. 1995, 34, 2311-2327; b) T. Michinobu, C. Boudon, J.-P. Gisselbrecht, P. Seiler, B. Frank, N. N. P. Moonen, M. Gross, F. Diederich, Chem. Eur. J. 2006, 12, 1889-1905.

[11] a) T. Yokoyama, S. Yokoyama, T. Kamikado, Y. Okuno, S. Mashiko, Nature 2001, 413, 619-621; b) Y. Okuno, T. Yokoyama, S. Yokoyama, T. Kamikado, S. Mashiko, J. Am. Chem. Soc. 2002, 124, 7218-7225; c) N. Wintjes, D. Bonifazi, F. Cheng, A. Kiebele, M. Stöhr, T. Jung, H. Spillmann, F. Diederich, Angew. Chem. 2007, 119, 4167-4170; Angew. Chem. Int. Ed. 2007, 46, 4089-4092; d) N. Wintjes, J. Hornung, J. LoboCheca, T. Voigt, T. Samuely, C. Thilgen, M. Stöhr, F. Diederich T. A. Jung, Chem. Eur. J. 2008, 14, 5794-5802; e) L. A. Fendt, M. Stöhr, N. Wintjes, M. Enache, T. A. Jung, F. Diederich, Chem. Eur. J. 2009, 15, 11139-11150.

[12] a) C. S. Wood, F. B. Mallory, J. Org. Chem. 1964, 29, 3373-3377; b) M. Flammang-Barbieux, J. Nasielski, R. H. Martin, Tetrahedron Lett. 1967, 8, 743-744; c) F. B. Mallory, C. W. Mallory, Org. React. 1984, 30, 1-456.

[13] R. G. Carlson, K. Srinivasachar, R. S. Givens, B. K. Matuszewski, J. Org. Chem. 1986, 51, 3978-3983.

[14] a) L. B. Liu, B. Yang, T. J. Katz, M. K. Poindexter, J. Org. Chem. 1991, 56, 3769-3775; b) M. Gingras, C. Collet, Synlett 2005, 2337-2341; c) E. Murguly, R. McDonald, N. R. Branda, Org. Lett. 2000, 2, 3169-3172.

[15] E. Vowinkel, J. Bartel, Chem. Ber. 1974, 107, 1221-1227.

[16] J. Chen, Y. Zhang, L. Yang, X. Zhang, J. Liu, L. Li, H. Zhang, Tetrahedron 2007, 63, 4266-4270.

[17] a) S. Grimme, J. Harren, A. Sobanski, F. Vögtle, Eur. J. Org. Chem. 1998, $1491-1509$; b) F. Furche, R. Ahlrichs, C. Wachsmann, E. Weber, A. Sobanski, F. Vögtle, S. Grimme, J. Am. Chem. Soc. 2000, 122, 1717-1724; c) T. Bürgi, A. Urakawa, B. Behzadi, K.-H. Ernst, A. Baiker, New J. Chem. 2004, 28, $332-$ 334.

[18] M. Taniguchi, H. Nakagawa, A. Yamagishi, K. Yamada, J. Mol. Catal. A 2003, 199, 65-71.

[19] K. H. Ernst, M. Neuber, M. Grunze, U. Ellerbeck, J. Am. Chem. Soc. 2001, 123, 493-495.

[20] P. Rahe, M. Nimmrich, A. Greuling, J. Schütte, I. G. Stará, J. Rybáček, G. Huerta-Angeles, I. Starý, M. Rohlfing, A. Kühnle, J. Phys. Chem. C 2010, 114, 1547-1552.

[21] M. Parschau, R. Fasel, K.-H. Ernst, Cryst. Growth Des. 2008, 8, $1890-1896$.

[22] S. Grimme, J. Comput. Chem. 2006, 27, 1787.

[23] N. Atodiresei, V. Caciuc, J.-H. Franke, S. Blügel, Phys. Rev. B 2008, 78, 045411 . 


\section{Communications}

\section{Communications}

Two-Dimensional Sbontaneous Resolution

M. Stöhr,* S. Boz, M. Schär,

M.-T. Nguyen, C. A. Pignedoli,

D. Passerone,* W. B. Schweizer,

C. Thilgen, T. A. Jung,*

F. Diederich*

IIII-III

Self-Assembly and Two-Dimensional Spontaneous Resolution of CyanoFunctionalized [7]Helicenes on $\mathrm{Cu}(111)$

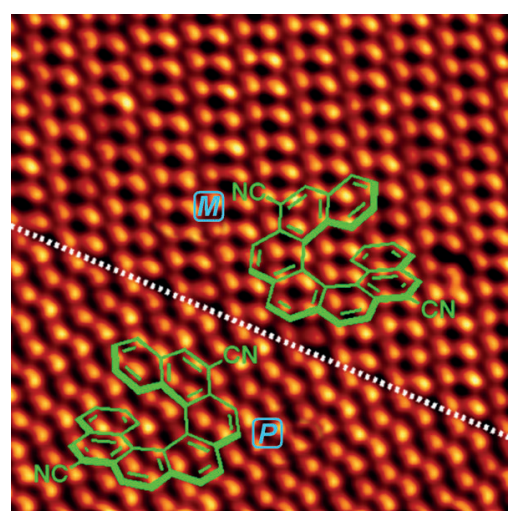

Birds of a feather flock together. STM and DFT studies provide the first example of spontaneous chiral resolution of a helicene on a surface. Racemic 6,13-dicyano[7]helicene forms fully segregated domains of pure enantiomers (2D conglomerate) on $\mathrm{Cu}(111)$. The propensity of the system to optimize intermolecular $\mathrm{CN}$...HC(Ar) hydrogen bonding and $\mathrm{CN}$... CN dipolar interactions translates into chiral recognition with preferential assembly of homochiral molecules. 